\title{
Consistência das Estratégias de Instituições de Ensino Superior: Um Estudo baseado na Percepção dos Stakeholders utilizando-se do Balanced Scorecard
}

\begin{abstract}
Resumo
A orientação estratégica da empresa foi concebida como uma ferramenta de gestão conhecida como o Balanced Scorecard (BSC), que objetiva medir e monitorar a estratégia em ação. Este trabalho teve como objetivo verificar a consistência estratégica na percepção dos stakeholders nas Instituições de Ensino Superior (IES) privadas, por meio das perspectivas do Balanced Scorecard. A metodologia utilizada foi uma pesquisa descritiva, por meio de uma abordagem quantitativa e com coleta de dados por meio de questionário aplicado em quatro IES no Estado de Minas Gerais, com diretores/coordenadores, professores e alunos denominados de stakeholders, para identificar, a partir de um modelo de Balanced Scorecard com quatro indicadores em cada perspectiva (financeira, clientes, aprendizagem e crescimento e processos internos), a consistência das estratégias percebida por esses grupos. Os principais resultados apontaram para uma diferença de percepção dos gestores quanto às perspectivas, com um grau de importância maior dado à perspectiva "Aprendizagem e Crescimento" e "Processos Internos". O grupo de professores foi o que atribuiu menos importância para a perspectiva "Clientes". As principais inconsistências encontradas foram na perspectiva "Processos Internos". A perspectiva "Financeira" foi a que apresentou menos gaps, quando comparada entre os grupos, o que revela uma inconsistência estratégica nas IES por meio da percepção dos stakeholders. Conclui-se que a consistência estratégica pode contribuir com a competitividade organizacional, identificando a existência de alinhamento nas ações desenvolvidas que resultam em maior eficiência para um cenário competitivo de acordo com seus interessados.
\end{abstract}

Palavras Chave: Consistência Estratégica. Balanced Scorecard. Stakeholders.

\begin{abstract}
Alexsandra Barcelos Dias
Mestre em Ciências Contábeis pela Fucape Business School e Coordenadora de Curso da Universidade Presidente Antônio Carlos, (Unipac GV). Contato: Rua Manoel Byrro - até n. ${ }^{\circ} 499 / 500$. Lourdes. Governador Valadares (MG), CEP: 35.032-620 E-mail: alexsandrabarcelosdias@hotmail.com
\end{abstract}

Valquíria Aparecida dos Santos Mestre em Ciências Contábeis pela Fucape Business School e professora na Escola Superior Aberta do Brasil. Contato: Av. Santa Leopoldina, n. $^{\circ} 840$. Coqueiral de Itaparica. Vila Velha - ES, CEP: 29.1619-65 E-mail: valquiriaasantos@gmail.com

\section{Aziz Xavier Beiruth}

Doutor em Controladoria e Contabilidade pela USP e professor da Fucape Business School. Contato: Av. Fernando Ferrari, . $^{0}$ 1358. Boa Vista. Vitória (ES). CEP: 29.075505.E-mail: aziz@fucape.br 


\section{Introdução}

Com a expansão das Instituições de Ensino Superior privadas (IES) brasileiras, além de vários fatores como a internacionalização, as fusões, incorporações e cisões, processo tecnológico e as mudanças de comportamento do consumidor, as instituições buscam padrões de eficiência para sua gestão. Para que as IES possam conduzir suas ações de forma competitiva, é necessário o uso da gestão estratégica. A gestão estratégica é uma vantagem competitiva que busca identificar as melhores práticas e estratégias para orientação dos negócios e avaliar constantemente a consistência de suas ações (Chen, Delmas \& Lieberman, 2015).

Para se avaliar constantemente a consistência de suas ações, as empresas adotam a consistência estratégica, que pode ser definida como uma sequência de decisões de alocação de recursos internamente consistentes e condicionais que são projetados para cumprir os objetivos de uma organização, compartilhando os recursos de acordo com os interesses das unidades institucionais, levando ao desempenho dos negócios (Venkatraman \& Camillus, 1984).

A medição de desempenho aliada à orientação estratégica da empresa foi concebida como uma ferramenta de gestão conhecida como Balanced Scorecard (BSC), que objetiva medir e monitorar a estratégia em ação. Esse instrumento é organizado em quatro perspectivas distintas: "Financeira", "Cliente", "Processo Interno" e "Aprendizado e Crescimento", e é utilizado para um dos objetivos centrais do BSC, que é comunicar a estratégia em todas as áreas da empresa, buscando produzir uma visão unificada da empresa por meio dos indicadores de desempenho e da cadeia de causa e efeito. O BSC foi desenvolvido pelo professor Robert S. Kaplan, da Harvard Business School, e o consultor David P. Norton, CEO do Instituto Nolan Norton no ano de 1992 (Hoque, 2014). De acordo com Frezatti, Bido, Cruz e Machado (2015), o BSC constitui um dos elementos relevantes para formalizar a implementação de estratégias.

A consistência estratégica dentro de uma instituição superior pode ser considerada como medida de verificação de cumprimento de sua missão e estratégia e apresentar uma relação com a percepção dos alunos, cujos os valores, missão, visão e estratégias foram bem definidos (Kettunem, 2003). O uso estratégico de ações na educação e a consistência dessas estratégias, utilizadas de forma eficiente, juntamente com os aspectos motivacionais, contribuem para um bom resultado e o sucesso dos estudantes (Meneghetti, De Beni \& Cornoldi, 2007).

Nesse contexto, este trabalho apresenta uma investigação da percepção entre os grupos diretores/ coordenadores, professores e alunos, tratados como stakeholders, da consistência estratégica de Instituições de Ensino Superior (IES) privadas, utilizando as perspectivas do BSC. Com isso, o presente estudo tem como objetivo responder ao seguinte questionamento: Existe consistência estratégica na percepção dos stakeholders nas Instituições de Ensino Superior Privadas por meio das perspectivas do Balanced Scorecard?

Dessa forma, este estudo tem como objetivo geral verificar a consistência estratégica na percepção dos stakeholders nas Instituições de Ensino Superior privadas, por meio das perspectivas do Balanced Scorecard.

Gerir uma IES é uma tarefa cada vez mais complexa, à medida que se multiplicam as exigências do mercado que buscam maior rentabilidade nas instituições, melhoria na qualidade do ensino e uma maior flexibilidade para assegurar a competitividade diante das necessidades impostas pelo mercado e pelo Ministério da Educação e Cultura (MEC). Ainda há a necessidade de se considerar em suas ações estratégias a crise financeira global e interna, que força as IES a competirem entre si em busca de mais alunos, o que, por sua vez, ressalta a questão da necessidade de uma administração mais estratégica e eficiente (Lira, Gonçalves \& Marques, 2015).

Nesse cenário, este trabalho pretende contribuir para a pesquisa de gestão em IES, investigando a consistência estratégica, que é vista como o compartilhamento de recursos e ações por unidade de negócios, prevendo o nível de desempenho de negócios que podem interferir nos resultados aos alunos, professores e interessados, propondo contribuir para o alinhamento das estratégias e redução dos gaps encontrados. Busca-se aprofundar o estudo de Rocha e de Oliveira Casartelli (2014), que destacam a importância do planejamento estratégico comum, a necessidade dos stakeholders para a disseminação da metodologia BSC e a participação ativa da alta administração. 
Diante do exposto, esta pesquisa busca contribuir com os achados de Rocha e de Oliveira Casartelli (2014) e Ferreira (2013), investigando a consistência estratégica em IES privadas, e estudar a percepção dos stakeholders, preenchendo, assim, a lacuna ainda existente, de forma a verificar como se ter uma vantagem competitiva e aprofundar na gestão estratégica em longo prazo (Teixeira \& Castro, 2015). Os autores destacam que, apesar da ampla utilização do BSC para o ensino superior, nenhum dos estudos mencionados ilustra um passo claro, para execução da etapa de estratégias.

A metodologia proposta é de caráter descritiva, sob uma abordagem quantitativa e com coleta de dados por meio de questionário aplicado em quatro Instituições de Ensino Superior privadas - Unipac situadas em Governador Valadares, Timóteo, Ipatinga e Betim, todas em Minas Gerais.

\section{Referencial Teórico}

\subsection{Consistência Estratégica}

Embora o conceito geral de consistência tenha sido utilizado com significados muito diferentes em uma variedade de contextos, Venkatraman e Camillus (1984) entende a sua utilização a partir de duas definições: (a) descritiva ou normativa e (b) conceitual ou empírica. Ele descreve que as pesquisas sobre estratégia envolvem o conceito geral de consistência, contingência, congruência, alinhamento, etc., invocando e testando várias relações teóricas, mas essas invocações variam significativamente na sua base teórica e quanto à sua precisão.

Um alto nível de consistência estratégica pode sinalizar a existência de uma estratégia competitiva forte, ou simplesmente inércia estrutural (Porter, 1992). A consistência estratégica significa um equilíbrio na alocação de recursos nas empresas. A consistência também pode ser entendida como um equilíbrio entre as escolhas estratégicas em negócios e os níveis funcionais dessas estratégias (Harrison, Hall \& Nargundakar, 1993).

O estudo de Lamberg, Tikkamen, Nokelainen e Suur-Inkeroinen (2009) se baseou na consistência estratégica conforme o padrão de competitividade das ações e descreve que a consistência estratégica se refere à comparabilidade no repertório e na quantidade de ações que uma organização compromete-se para a condução de sua posição competitiva. Assim, um alto nível de consistência estratégica pode sinalizar a existência de uma explícita ou implícita estratégia competitiva.

Ao relacionar a consistência estratégica, por meio do processo da gestão do BSC na IES, destaca-se como um ponto forte o reconhecimento da importância do planejamento estratégico para as IES. Outro ponto seria a existência de um foco estratégico comum, assim como a definição de prioridades institucionais abrangentes, a criação de uma equipe de implantação capaz de disseminar a metodologia do BSC e a obtenção uma ferramenta da TI para apoiar o processo e contar a participação ativa da alta administração nesse processo (Rocha \& de Oliveira Casartelli, 2014).

\subsection{Stakeholders}

Os stakeholders, em um espaço social da organização de forma restrita, podem ser entendidos como qualquer grupo ou indivíduo que possa afetar ou ser afetado pelos objetivos organizacionais (Freeman, 2010). Starik (1994), anos depois, considera-os em função da extensão, do tipo de benefício, da importância e, principalmente, da percepção do que sejam as organizações e quais seus objetivos.

Os stakeholders são classificados como partes interessadas, internas e externas, e devem atuar com responsabilidade corporativa social, agilidade e desempenho (Clarkson, 1995), podendo ser divididos em dois grupos: os interessados ambientais (clientes, proprietários e comunidade) e atores do processo (funcionários e fornecedores). Os stakeholders são considerados partes interessadas que contribuem com o gerenciamento e avaliação organizacional (Atkinson, Waterhouse \& Wells. 1997) 
Dentro dessa diversidade de considerações e proposições, a literatura considera stakeholders os empregados, os acionistas, os fornecedores, os clientes e a comunidade (Moore, 1999). Nesta pesquisa trabalhou-se com os seguintes stakeholders: alunos, professores, coordenadores e diretores.

\subsection{Estratégias e Gestão das Instituições de Ensino Superior}

Para estabelecer uma gestão eficiente nas Instituições de Ensino Superior é necessário considerar alguns valores, que pode ser por meio da criação de valor para alunos, professores, funcionários e sociedade, fundamentando-se no gerenciamento equilibrado dos ativos intangíveis e no planejamento e controle de recursos financeiros que se traduzirão em excelência na prestação de serviços educacionais e na perenidade financeira do negócio (Silva, 2009). Para gerenciar esses valores, pode-se utilizar o BSC, que está voltado para o futuro, visando o melhoramento interno, o investimento nos funcionários e no desempenho em longo prazo (Kaplan \& Norton, 1997).

Rocha e de Oliveira Casartelli (2014) pressupõem que, com a adequação do BSC para as Instituições de Ensino Superior, é possível criar e comunicar a estratégia e as direções, por meio de um sistema de indicadores, para todos os níveis hierárquicos da organização, levando à consistência estratégica. Sendo assim, é possível adotar o BSC no sistema de medição e avaliação de desempenho ligado à estratégia organizacional, oferecendo aos gestores das Instituições de Ensino Superior opções viáveis para decidir seus planos e ações.

O BSC é uma ferramenta que foi desenvolvida nos Estados Unidos, no ano de 1992, e tem como principais autores/divulgadores Robert Kaplan e David Norton. Um dos principais objetivos do Balanced Scorecard é permitir aos gestores o monitoramento e ajuste de implementação de estratégias organizacionais (Kaplan \& Norton, 1997). A proposta dos autores por meio do desenvolvimento do Balanced Scorecard, é que as organizações além de mensurarem os indicadores financeiros também o fizessem com os não financeiros, como clientes e conhecimento de seus colaboradores, sempre de forma a criar valor no processo, produto e/ou serviço. De acordo com Melo Santos, Bronzo, Olieira e Resende (2014), o modelo do BSC integra medidas para as estratégias de negócio, de acordo com Kaplan em pelo menos quatro dimensões: "Financeira", "Cliente, "Processos Internos" e "Conhecimento e Crescimento".

O BSC complementa as medidas financeiras do desempenho passado com medidas dos vetores que impulsionam o desempenho futuro (Kaplan \& Norton, 1997). Em seu estudo, Lima Júnior (2008) afirma que o BSC evoluiu a partir de uma ferramenta de medição para se tornar aquilo que Kaplan e Norton (1997) descrevem como sendo um sistema de gerenciamento estratégico. Embora a intenção original do sistema Scorecard fosse equilibrar os números históricos financeiros com as diretrizes do valor futuro para a organização, à medida que mais empresas testaram o conceito, elas descobriram que o Balanced Scorecard, com sua estratégia, é uma ferramenta essencial para o alinhamento em ações de curto prazo.

Os objetivos principais do BSC, segundo Kaplan e Norton (1997), são: esclarecer e traduzir a visão e a estratégia; comunicar e associar objetivos e medidas estratégicas; planejar, estabelecer metas e alinhar iniciativas estratégicas; e melhorar o feedback e o aprendizado estratégico. Acrescentam ainda que o BSC não pode ser entendido apenas como conjunto de indicadores críticos ou fatores chaves de sucesso, mas sim como a composição de uma cadeia articulada de medidas e objetivos lógicos que se reforcem reciprocamente, que funcionem como um conjunto complexo de relações de causa e efeito (devem permear todas as quatro perspectivas) e que seja possível a combinação de medidas de resultados e vetores de desempenho (indicações quantitativas de tempo ou porcentagem), pois as medidas de resultados sem os vetores de desempenho não comunicam a forma que os resultados são alcançados (Kaplan \& Norton, 1997).

As Instituições de Ensino Superior precisam, como qualquer outra organização, ter sustentabilidade financeira, satisfação de clientes e alunos, aprendizado e crescimento do seu capital humano e processos internos que a conduzam a uma gestão focada na eficiência. Essa metodologia de gestão nas IES pode ocorrer por meio da adaptação das quatro perspectivas do BSC, pela criação de mapas estratégicos, objetivos estratégicos e, por fim, indicadores de resultados inerentes ao setor (Silva, 2009). 
Rocha e de Oliveira Casartelli (2014) discorrem que a utilização do BSC nas IES privadas contribui para a definição, classificação e comunicação de sua estratrégia, traduzindo a visão e a estratégia em um conjunto de medidas de desempenho. As perspectivas do Balanced Scorecard nas Instituições de Ensino Superior foram sintetizadas por eles em: responsabilidade financeira, clientes, educação, processos internos, aprendizagem e crescimento.

O BSC foi estudado por Rüdell Bolignon, Pentiado Godoy e Souto Bolzan Medeiros (2014), que propuseram identificar os passos necessários para a implantação da ferramenta de avaliação de desempenho organizacional. O objetivo principal do trabalho dos autores era traduzir a visão e a missão de uma organização em um conjunto de indicadores de desempenho, com base na metodologia do Balanced Scorecard, para a gestão estratégica da organização. E identificou-se que os principais benefícios do BSC são: um maior conhecimento da estratégia e missão organizacional, motivação dos colaboradores, integração das diferentes áreas de atuação e uma melhoria no acompanhamento das ações estratégicas e na visão abrangente do desempenho organizacional.

Para a aplicação do questionário, foram avaliados indicadores de desempenho e adotados os três princípios do BSC: a relação de causa e efeito; os resultados e vetores de desempenho; e a relação com os fatores financeiros, conforme proposto por Silva (2009), dentro das perspectivas adaptadas do autor de Ferreira (2013), sendo: Aprendizado e Crescimento; qualificação dos professores; qualificação dos técnicos administrativos; motivação e satisfação no trabalho do corpo de professores; e aprimoramento em Tecnologia da Informação - TI. Os três principios são:

a) Processos Internos: projeto pedagógico/diversidade de cursos/qualidade de ensino; programa de pesquisa e extensão; infraestrutura física e tecnológica (acervo bibliográfico, laboratórios, localização, etc.); e projetos e programas sociais;

b) Clientes: reputação pela qualidade de ensino; atendimento às expectativas do aluno; criação de valor ao aluno por meio do serviço de apoio e tradição da marca reconhecida pelo mercado;

c) Financeira: sustentabilidade da instituição; relação custo/benefício da mensalidade; utilização racional dos recursos disponíveis; e programas de incentivos (bolsas/políticas de financiamentos/convênios e parcerias).

As hipóteses a serem testadas neste estudo foram elaboradas com base na revisão da literatura, especialmente a partir do resultado de pesquisa de Kaplan e Norton (1997), Serdar Asan e Tanyas (2007) - Lima Júnior (2008), Silva (2009), Lamberg et. al. (2009), Rocha (2000), Ferreira (2013) e Rocha e de Oliveira Casartelli (2014).

Assim, o estudo pretende testar as seguintes hipóteses:,

$\mathrm{H}_{0}$ : Não existem diferenças estatisticamente significantes entre os scores médios obtidos para as variáveis, considerando os três grupos direção/coordenação, professores e alunos.

$\mathrm{H}_{1}$ : Existem diferenças estatisticamente significantes entre os scores médios obtidos para as variáveis, considerando os três grupos direção/coordenação, professores e alunos.

\section{Metodologia da Pesquisa}

A metodologia proposta é de caráter descritiva, por meio de uma abordagem quantitativa e com coleta de dados por meio de questionário em quatro Instituições de Ensino Superior privadas - Unipac situadas em Governador Valadares, Timóteo, Ipatinga e Betim.

A pesquisa se desenvolveu por intermédio de levantamento de coleta de dados survey e foi realizada por meio de questionários, com a direção/coordenação, professores e alunos para avaliar a consistência estratégica entre esses sujeitos dentro das perspectivas do BSC. 
Na primeira fase da pesquisa, foi feita a coleta de dados em uma amostra piloto com duzentos alunos, 21 (vinte e um) diretores/coordenadores e 31 (trinta e um) professores para validar o instrumento de pesquisa.

$\mathrm{Na}$ segunda fase da pesquisa, foi feita a coleta de novos dados, não sendo reaproveitados os da amostra piloto, com 903 (novecentos e três) alunos, 37 (trinta e sete) diretores/coordenadores e 90 (noventa) professores.

$\mathrm{Na}$ avaliação dos indicadores de desempenho, foram adotados os três princípios do Balanced Scorecard: a relação de causa e efeito; resultados e vetores de desempenho; e a relação com os fatores financeiros, conforme proposto por Silva (2009), de acordo com o questionário adaptado de Ferreira (2013).

\subsection{Instituições das Análises de Dados e População}

A população da pesquisa é composta por pessoas da coordenação/direção, professores e alunos dos cursos de graduação em Ciências Contábeis em um total de 45 pessoas no grupo da coordenação/direção, 322 pessoas no grupo professores e 8.520 alunos, aproximadamente (dados referentes ao ano de 2011).

Foi elaborado um questionário que abrange quatro perspectivas do BSC: "Aprendizagem e Crescimento", "Processos Internos", "Clientes e Financeiras", em que o respondente atribui uma nota de 1 a 5 que vai de nenhuma importância a importância total. Busca-se com essas perspectivas do BSC considera o grau de importância na opinião do respondente, de cada indicador na formação do padrão de qualidade da Unipac, totalizando 16 abordagens.

Foi realizada uma pesquisa com 1.030 respondentes, sendo 31 eliminados por apresentar variância zero, totalizando 999 questionários válidos. Como proposto por Lima Júnior (2008), os questionários dos respondentes que apresentaram variância zero, ou seja, responderam para todos os indicadores o mesmo valor, foram eliminados das análises. Assim acredita-se que o respondente não ponderou ao responder, pois, em 16 itens, apresentou a mesma resposta. $\mathrm{Na}$ análise descritiva, a média representa o grau de importância dado pelos respondentes de acordo com o questionário realizado, que varia de 0 a 5 .

O gráfico de Radar foi utilizado para mostrar os resultados apresentados na análise descritiva. Esse gráfico, também conhecido como gráfico de Aranha ou gráfico de Estrela devido à sua aparência, plota os valores de cada categoria ao longo de um eixo separado que inicia no centro do gráfico e termina no anel externo. O gráfico de Radar compara os valores agregados de várias sequências de dados.

Para verificar se existem diferenças significativas visualizadas por intermédio da análise descritiva dos dados, foi realizada a Análise de Variância (Anova). Pela análise de variância é possível avaliar se as diferenças observadas entre mais de duas médias amostrais podem ser distribuídas ao acaso, ou se de fato existem diferenças entre as médias das populações correspondentes (Werkema \& Aguiar, 1996). Para comparar as médias dos indicadores do Balanced Scorecard entre os grupos, foi realizado inicialmente o teste de Levene, identificando a homogeneidade de variância entre os grupos. Esse teste permite averiguar a homogeneidade das variâncias a serem comparadas. E, neste caso, se o valor de $p$ for maior que 0,05 indica que as variâncias são significativamente homogêneas.

Observando-se diferenças entre as médias, ao tentar descobrir para quais grupos as médias são diferentes, foi realizado o teste de Games-Howell. O teste de comparação de Games-Howell é indicado para situações nas quais as variâncias não são as mesmas entre os grupos e para quando os grupos possuem tamanhos muito diferentes (SPSS INC, 2003).

Quando não foi rejeitada a hipótese de igualdade de variâncias, utilizou-se o teste de Scheffe. O teste de Scheffe é robusto para casos em que as amostras possuem tamanhos muito diferentes (SPSS INC, 2003). A Análise Fatorial tem como objetivo analisar as inter-relações entre um vasto número de variáveis, identificando um grupo de dimensões latentes comuns, as quais se denominam fatores (Hair, Babin, Money \& Samouel, 2005). 


\section{Análise de Resultados}

O público-alvo da pesquisa está dividido em três grupos de análise, sendo eles "Diretor/Coordenador", "Professores" e "Alunos". A Figura 1 apresenta a distribuição da amostra por grupos. No total, foram realizadas (válidas) 999 entrevistas, das quais 3,7\% (37 entrevistados) são do grupo Diretor/Coordenador; 9,0\% (90 entrevistados), do grupo "Professores"; e 87,3\% (872 entrevistados), do grupo "Alunos". Todas as análises foram realizadas para os três grupos de investigação.

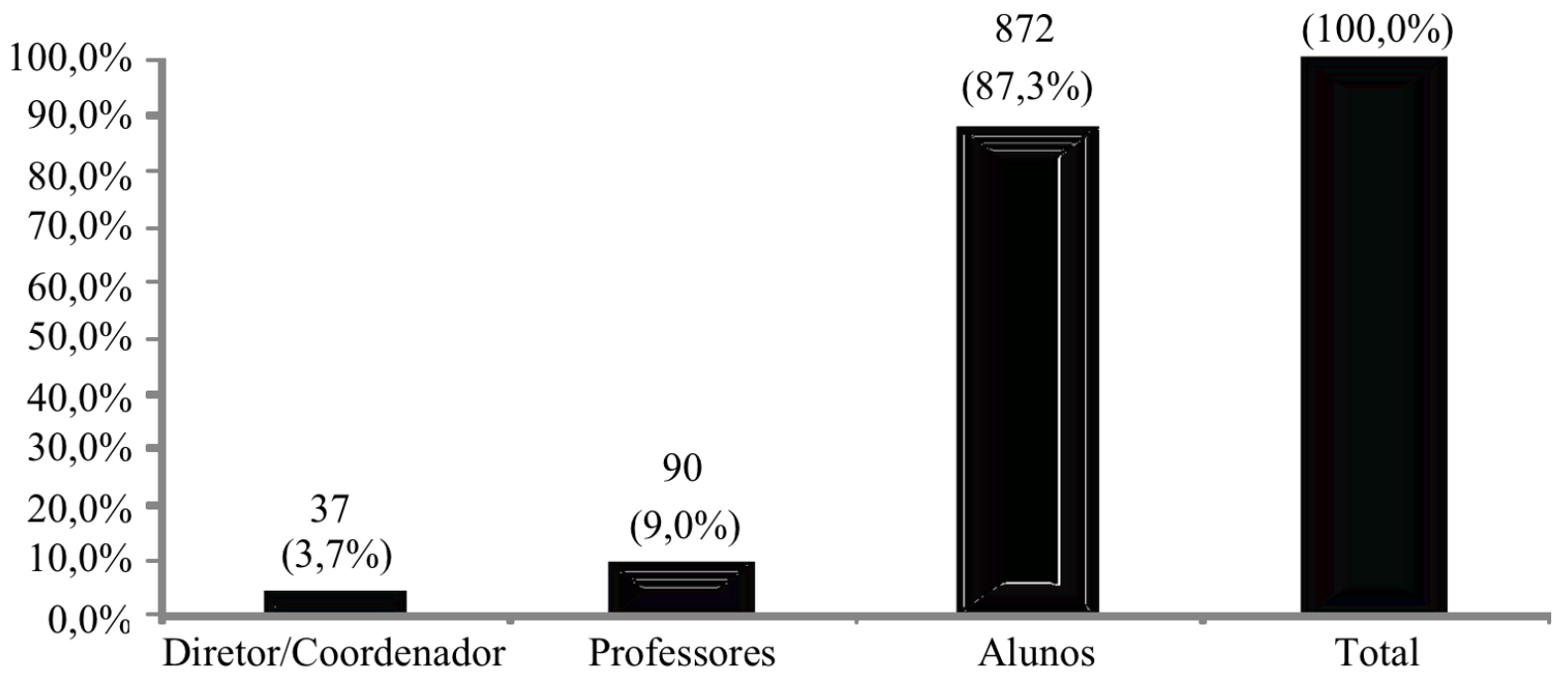

Figura 1. Distribuição dos entrevistados por grupos de análise

Fonte: dados da pesquisa.

\subsection{Análises Descritivas dos Graus de Importância dos Indicadores}

Para cada um dos indicadores, foram calculadas algumas estatísticas descritivas como: a média, o desvio-padrão e o grau de importância, que é a soma dos percentuais das notas 4 (quatro) e 5 (cinco) da escala de notas utilizada no questionário. A média geral dos indicadores foi calculada, primeiramente, obtendo-se média das notas de cada entrevistado para os indicadores. Posteriormente, foi feita a média entre os entrevistados e calculado o desvio-padrão da média geral, em cada perspectiva. A significância das diferenças entre as médias obtidas foram avaliadas por meio de testes estatísticos de Análise de Variâncias (Anova), para comparação entre as médias das notas.

\subsubsection{Perspectiva aprendizagem e crescimento}

A primeira perspectiva do BSC avaliada foi referente à Aprendizagem e Crescimento. Observou-se que a significância das diferenças entre os grupos em relação aos indicadores que compõem as perspectivas tendem a ser bastante influenciadas pelo grupo "Alunos". A Tabela 1 apresenta algumas estatísticas descritivas. 
Tabela 1

Estatística Descritiva da Perspectiva Aprendizagem e Crescimento

\begin{tabular}{|c|c|c|c|c|c|}
\hline Grupos & Indicadores & Média & Desvio- Padrão & CV(\%) & $\begin{array}{l}\text { Grau de } \\
\text { Importância }\end{array}$ \\
\hline \multirow{5}{*}{ 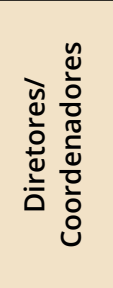 } & 1-Qualificação dos professores & 4,84 & 0,44 & $9,09 \%$ & $97,30 \%$ \\
\hline & 2-Qualificação dos técnicos administrativos & 4,57 & 0,60 & $13,13 \%$ & $94,59 \%$ \\
\hline & $\begin{array}{l}\text { 3-Motivação e satisfação no trabalho do corpo } \\
\text { docente }\end{array}$ & 4,84 & 0,44 & $9,09 \%$ & $97,30 \%$ \\
\hline & 4-Aprimoramento em Tecnologia da Informação (TI) & 4,27 & 0,84 & $19,67 \%$ & $83,78 \%$ \\
\hline & Média Geral & 4,62 & 0,36 & $7,79 \%$ & $93,24 \%$ \\
\hline \multirow{5}{*}{ 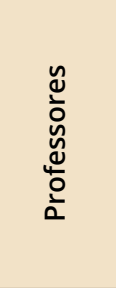 } & 1-Qualificação dos professores & 4,66 & 0,72 & $15,45 \%$ & $93,33 \%$ \\
\hline & 2-Qualificação dos técnicos administrativos & 4,32 & 0,83 & $19,21 \%$ & $86,67 \%$ \\
\hline & $\begin{array}{l}\text { 3-Motivação e satisfação no trabalho do corpo } \\
\text { docente }\end{array}$ & 4,67 & 0,67 & $14,35 \%$ & $94,44 \%$ \\
\hline & 4-Aprimoramento em Tecnologia da Informação (TI) & 4,14 & 0,80 & $19,32 \%$ & $80,00 \%$ \\
\hline & Média Geral & 4,45 & 0,55 & $12,36 \%$ & $88,61 \%$ \\
\hline \multirow{5}{*}{$\begin{array}{l}\tilde{n} \\
\frac{0}{3} \\
\frac{3}{<}\end{array}$} & 1-Qualificação dos professores & 4,56 & 0,72 & $15,79 \%$ & $90,25 \%$ \\
\hline & 2-Qualificação dos técnicos administrativos & 4,09 & 0,92 & $22,49 \%$ & $74,66 \%$ \\
\hline & $\begin{array}{l}\text { 3-Motivação e satisfação no trabalho do corpo de } \\
\text { professores }\end{array}$ & 4,34 & 0,87 & $20,05 \%$ & $83,72 \%$ \\
\hline & 4-Aprimoramento em Tecnologia da Informação (TI) & 4,02 & 1,04 & $25,87 \%$ & $72,59 \%$ \\
\hline & Média Geral & 4,25 & 0,72 & $16,94 \%$ & $80,30 \%$ \\
\hline
\end{tabular}

Fonte: dados da pesquisa.

O grupo "Diretor/Coordenador" tende a dar notas mais altas aos indicadores referentes à "Aprendizagem e Crescimento", o que pode ser observado para os valores médios e para os graus de importância atribuídos e apresentados na Tabela 1 . O grupo alunos tende a atribuir menos importância a essa perspectiva em relação aos demais grupos, quando se observa os valores médios das notas. Outro fator relevante é em termos de homogeneidade dos grupos, pois o Coeficiente de Variação (CV) é menor para o grupo Diretor/Coordenador (7,79\%), o que indica que as percepções tendem a ser mais parecidas entre os entrevistados. Algumas discrepâncias nos resultados podem ser visualizadas na Figura 2. 


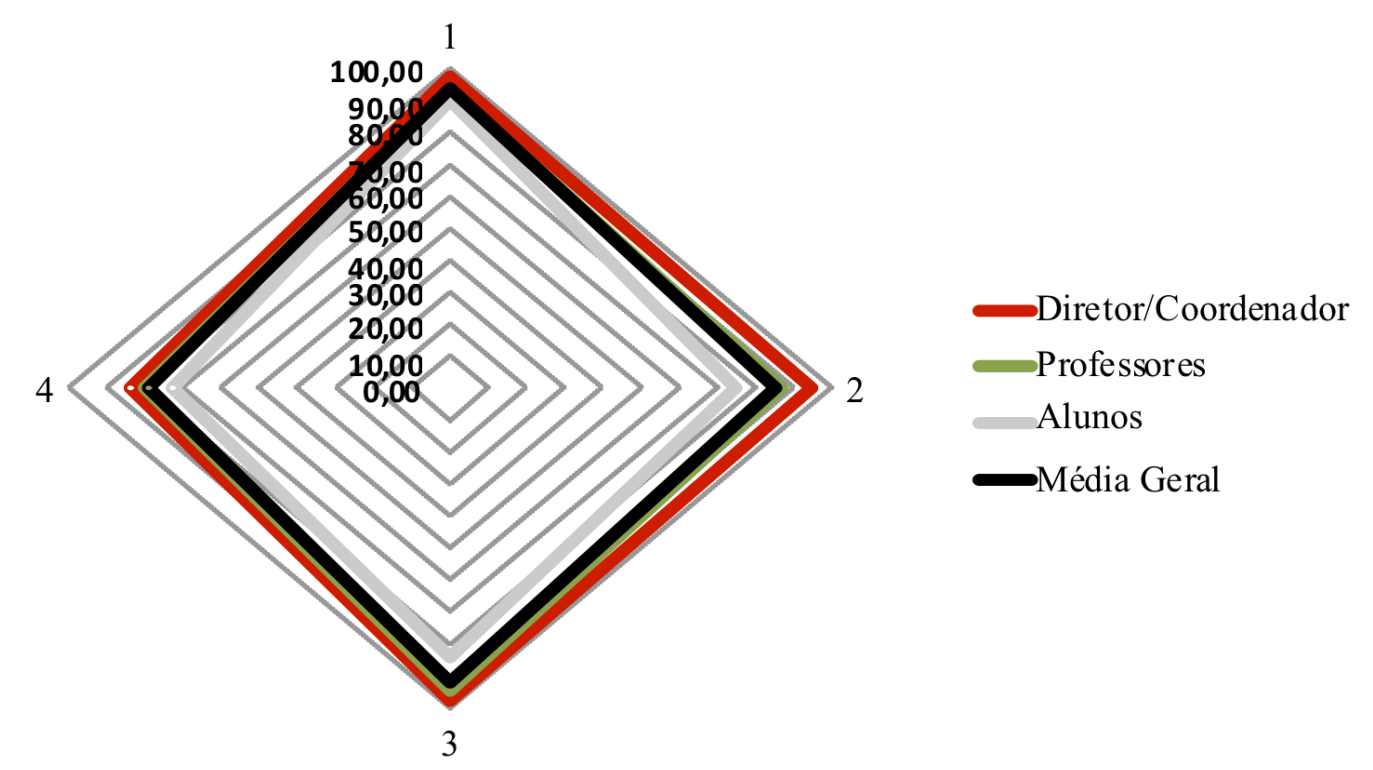

Legenda:

1. Qualificação dos professores

2. Qualificação dos técnicos administrativos

3. Motivação e satisfação no trabalho do corpo docente

4. Aprimoramento em Tecnologia da Informação TI

Figura 2. Grau de importância para os indicadores da perspectiva Aprendizagem e Crescimento

Fonte: dados da pesquisa

Pelo Gráfico 2, pode-se notar que o grau de importância atribuído pelos alunos a essa perspectiva tende a ser menor em comparação ao grupo dos professores e o grupo "Diretor/Coordenador", respectivamente, para todos os indicadores. Quanto mais ao extremo do gráfico de Radar, maior é o percentual do grau de importância atribuído aos indicadores. As linhas dos percentuais de notas dos alunos estão mais internas ao gráfico de Radar e inferiores à média geral, o que indica notas de importância mais baixas.

\subsubsection{Perspectiva processos internos}

A segunda perspectiva do Balanced Scorecard avaliada foi referente a "Processos Internos". Observou-se significância das diferenças entre os grupos em relação aos indicadores que compõem essa perspectiva. Essas diferenças estão relacionadas, principalmente, à avaliação dos diretores/coordenadores. A Tabela 2 apresenta as estatísticas descritivas.

O grupo "Diretor/Coordenador" tende a atribuir notas mais altas aos indicadores referentes à Perspectiva Processos Internos, o que pode ser observado para os valores médios e para os graus de importância atribuídos na Tabela 2. Os alunos tendem a atribuir menos importância a essa perspectiva em relação aos demais grupos quando se observa os valores médios das notas, embora para essa perspectiva as diferenças pareçam ser menores. Outro fator relevante é em termos de homogeneidade dos grupos. O Coeficiente de Variação (CV) é menor para o grupo "Diretor/Coordenador" (8,30\%) e para o grupo "Professores" (9,74\%), o que indica que as percepções tendem a ser mais parecidas entre os entrevistados desses dois grupos. Algumas discrepâncias nos resultados podem ser visualizadas na Figura 3. 
Tabela 2

Estatística Descritiva da Perspectiva Processos Internos

\begin{tabular}{|c|c|c|c|c|c|}
\hline Grupos & Indicadores & Média & Desvio-Padrão & CV(\%) & Grau de Importância \\
\hline \multirow{5}{*}{ 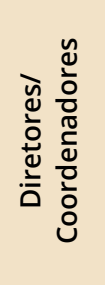 } & $\begin{array}{l}\text { 1-Projeto pedagógico/diversidade de } \\
\text { cursos/qualidade de ensino }\end{array}$ & 4,76 & 0,50 & $10,50 \%$ & $97,30 \%$ \\
\hline & 2-Programa de pesquisa e extensão & 4,24 & 0,55 & $12,97 \%$ & $94,59 \%$ \\
\hline & 3-Infraestrutura física e tecnológica & 4,73 & 0,45 & $9,51 \%$ & $100,00 \%$ \\
\hline & 4-Projetos e programas sociais & 4,11 & 0,70 & $17,03 \%$ & $81,08 \%$ \\
\hline & Média Geral & 4,46 & 0,37 & $8,30 \%$ & $93,24 \%$ \\
\hline \multirow{5}{*}{ 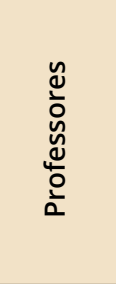 } & $\begin{array}{l}\text { 1-Projeto pedagógico/diversidade de } \\
\text { cursos/qualidade de ensino }\end{array}$ & 4,66 & 0,56 & $12,02 \%$ & $95,56 \%$ \\
\hline & 2-Programa de pesquisa e extensão & 4,31 & 0,71 & $16,47 \%$ & $87,78 \%$ \\
\hline & 3-Infraestrutura física e tecnológica & 4,50 & 0,66 & $14,67 \%$ & $91,11 \%$ \\
\hline & 4-Projetos e programas sociais & 3,84 & 0,91 & $23,70 \%$ & $58,89 \%$ \\
\hline & Média Geral & 4,33 & 0,41 & $9,47 \%$ & $83,33 \%$ \\
\hline \multirow{5}{*}{$\frac{n}{\frac{n}{2}}$} & $\begin{array}{l}\text { 1-Projeto pedagógico/diversidade de } \\
\text { cursos/qualidade de ensino }\end{array}$ & 4,34 & 0,89 & $20,51 \%$ & $83,94 \%$ \\
\hline & 2-Programa de pesquisa e extensão & 3,97 & 1,08 & $27,20 \%$ & $72,71 \%$ \\
\hline & 3-Infraestrutura física e tecnológica & 4,01 & 1,17 & $29,18 \%$ & $70,64 \%$ \\
\hline & 4-Projetos e programas sociais & 3,96 & 1,04 & $26,26 \%$ & $70,64 \%$ \\
\hline & Média Geral & 4,07 & 0,87 & $21,38 \%$ & $74,48 \%$ \\
\hline
\end{tabular}

Fonte: dados da pesquisa.

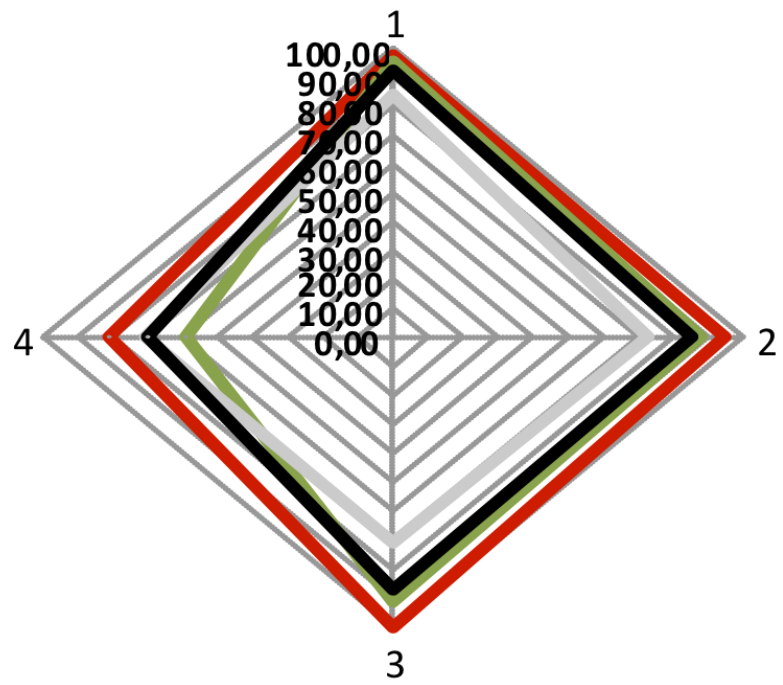

Diretor/Coordenador

Professores

Alunos

-Média Geral

Legenda:

1. Projeto pedagógico/diversidade de cursos/qualidade de ensino

2. Programa de pesquisa e extensão

3. Infraestrutura física e tecnológica

4. Projetos e programas sociais

Figura 3. Grau de importância para os indicadores da perspectiva Processos Internos Fonte: dados da pesquisa

Pela Figura 3, pode-se notar que o grau de importância atribuído pelos alunos essa perspectiva tende a ser menor em comparação ao grupo dos professores e ao grupo dos diretores/coordenadores, respectivamente, exceto para o atributo "Projetos e Programas Sociais". As linhas dos percentuais de notas dos alunos estão mais internas ao gráfico de Radar e inferiores à média geral, o que indica notas de importância mais baixas. 


\subsubsection{Perspectiva clientes}

A terceira perspectiva do Balanced Scorecard avaliada foi a de "Clientes". Observou-se significância das diferenças entre os grupos em relação aos indicadores que compõem essa perspectiva. Essas diferenças estão relacionadas principalmente à avaliação dos professores. A Tabela 5 apresenta as estatísticas descritivas.

Tabela 3

\section{Estatística Descritiva da Perspectiva Clientes}

\begin{tabular}{|c|c|c|c|c|c|}
\hline Grupos & Indicadores & Média & Desvio- Padrão & CV(\%) & $\begin{array}{c}\text { Grau de } \\
\text { Importância }\end{array}$ \\
\hline \multirow{5}{*}{ 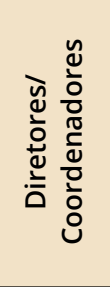 } & 1-Reputação pela qualidade de ensino & 4,95 & 0,33 & $6,67 \%$ & $100,00 \%$ \\
\hline & 2-Atender às expectativas do aluno & 4,08 & 0,86 & $21,08 \%$ & $81,08 \%$ \\
\hline & $\begin{array}{l}\text { 3-Atribuir valor ao aluno, por meio de serviços } \\
\text { de apoio }\end{array}$ & 3,86 & 0,92 & $23,83 \%$ & $78,38 \%$ \\
\hline & 4-Tradição da marca (reconhecida no mercado) & 4,30 & 1,13 & $26,28 \%$ & $78,38 \%$ \\
\hline & Média Geral & 4,30 & 0,55 & $12,79 \%$ & $84,46 \%$ \\
\hline \multirow{5}{*}{ 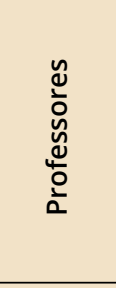 } & 1-Reputação pela qualidade de ensino & 4,62 & 0,59 & $12,77 \%$ & $94,44 \%$ \\
\hline & 2-Atender ás expectativas do aluno & 4,04 & 0,83 & $20,54 \%$ & $72,22 \%$ \\
\hline & $\begin{array}{l}\text { 3- Atribuir valor ao aluno, por meio de serviços } \\
\text { de apoio }\end{array}$ & 4,01 & 0,89 & $22,19 \%$ & $65,56 \%$ \\
\hline & 4-Tradição da marca (reconhecida no mercado) & 3,64 & 1,15 & $31,59 \%$ & $56,67 \%$ \\
\hline & Média Geral & 4,08 & 0,63 & $15,44 \%$ & $72,22 \%$ \\
\hline \multirow{5}{*}{$\begin{array}{l}\text { Oั } \\
\frac{5}{3}\end{array}$} & 1-Reputação pela qualidade de ensino & 4,31 & 0,92 & $21,35 \%$ & $81,88 \%$ \\
\hline & 2-Atender às expectativas do aluno & 4,23 & 1,02 & $24,11 \%$ & $80,96 \%$ \\
\hline & $\begin{array}{l}\text { 3- Atribuir valor ao aluno, por meio de serviços } \\
\text { de apoio }\end{array}$ & 4,10 & 1,04 & $25,37 \%$ & $75,23 \%$ \\
\hline & 4-Tradição da marca (reconhecida no mercado) & 4,10 & 0,99 & $24,15 \%$ & $75,80 \%$ \\
\hline & Média Geral & 4,19 & 0,82 & $19,57 \%$ & $78,47 \%$ \\
\hline
\end{tabular}

Fonte: dados da pesquisa.

Para a perspectiva "Clientes", o grupo "Professores" demonstra uma importância um pouco mais baixa em relação aos demais grupos. A Tabela 3 apresenta diferenças médias aparentemente significativas entre os grupos "Professores" e os demais grupos. O Coeficiente de Variação (CV) é menor para o grupo "Diretor/Coordenador" (12,79\%), o que indica que as percepções tendem a ser mais parecidas entre os entrevistados. Algumas discrepâncias nos resultados podem ser visualizadas no Figura 4. 


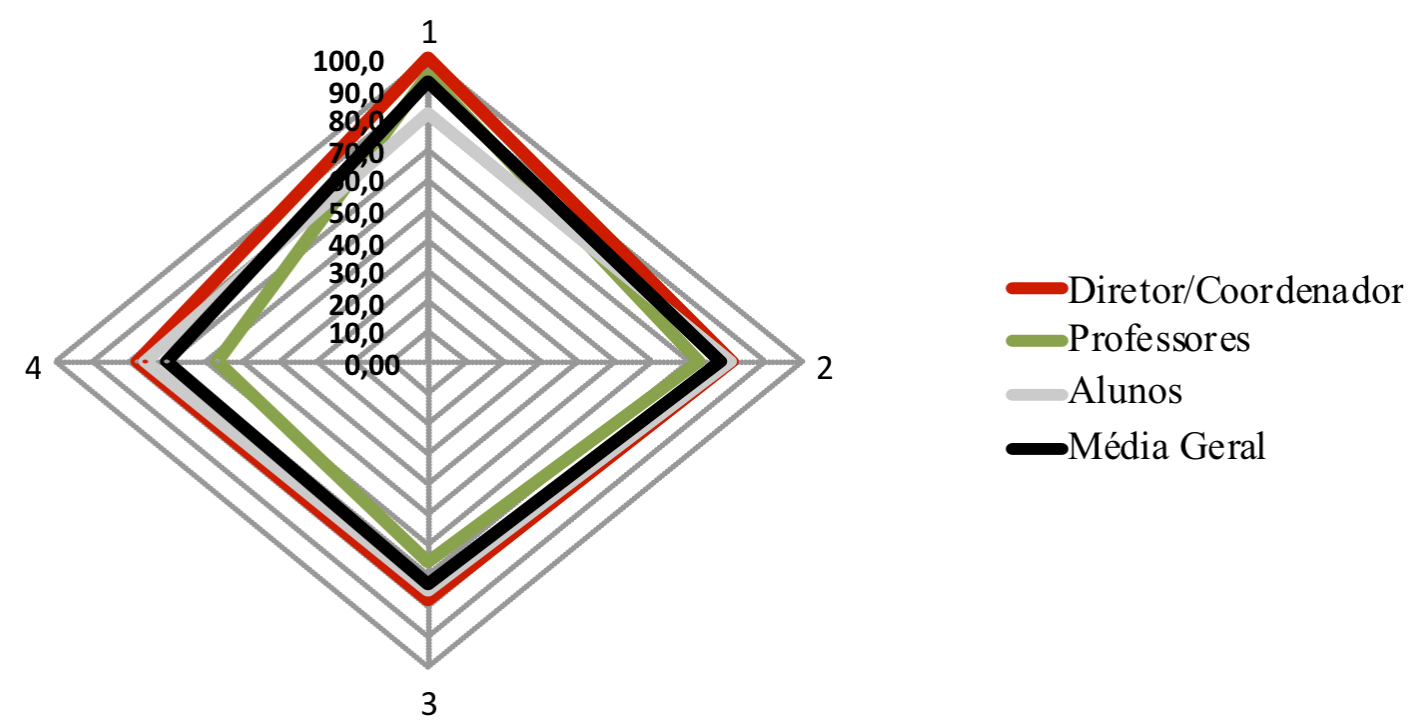

Legenda:

1. Reputação pela qualidade de ensino

2. Atender às expectativas do aluno

3. Atribuir valor ao aluno, por meio de serviços de apoio.

4. Tradição da marca reconhecida no mercado.

Figura 4. Grau de importância para os indicadores da perspectiva Clientes

Fonte: dados da pesquisa

Pela Figura 4, pode-se notar a baixa percepção dos professores em relação ao grau de importância para a perspectiva "Clientes". Para três dos quatro indicadores, as linhas referentes aos percentuais do grau de importância estão inferiores aos demais grupos, o que indica uma avaliação bastante negativa.

\subsubsection{Perspectiva financeira}

A última perspectiva do Balanced Scorecard avaliada foi a "Financeira". Observou-se significância das diferenças entre os grupos em relação aos indicadores que compõem essa perspectiva. A Tabela 4 apresenta as estatísticas descritivas. 
Tabela 4

\section{Estatística Descritiva da Perspectiva Financeira}

\begin{tabular}{|c|c|c|c|c|c|}
\hline Grupos & Indicadores & Média & Desvio- Padrão & CV(\%) & $\begin{array}{c}\text { Grau de } \\
\text { Importância }\end{array}$ \\
\hline \multirow{5}{*}{ 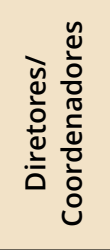 } & 1-Sustentabilidade financeira da instituição & 4,84 & 0,44 & $9,09 \%$ & $97,30 \%$ \\
\hline & 2-Recolocação custo/benefício da mensalidade & 4,35 & 0,68 & $15,63 \%$ & $89,19 \%$ \\
\hline & 3-Utilização racional dos recursos disponíveis & 4,27 & 0,73 & $17,10 \%$ & $83,78 \%$ \\
\hline & 4-Programas de incentivos & 3,65 & 0,86 & $23,56 \%$ & $62,16 \%$ \\
\hline & Média Geral & 4,28 & 0,49 & $11,45 \%$ & $83,11 \%$ \\
\hline \multirow{5}{*}{ 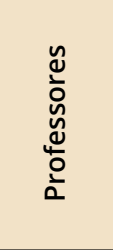 } & 1-Sustentabilidade financeira da instituição & 4,58 & 0,70 & $15,28 \%$ & $90,00 \%$ \\
\hline & 2-Recolocação custo/benefício da mensalidade & 4,06 & 0,83 & $20,44 \%$ & $74,44 \%$ \\
\hline & 3-Utilização racional dos recursos disponíveis & 4,09 & 0,76 & $18,58 \%$ & $75,56 \%$ \\
\hline & 4-Programas de incentivos & 3,91 & 1,02 & $26,09 \%$ & $65,56 \%$ \\
\hline & Média Geral & 4,16 & 0,55 & $13,22 \%$ & $76,39 \%$ \\
\hline \multirow{5}{*}{$\begin{array}{l}\stackrel{n}{\circ} \\
\stackrel{5}{\frac{1}{\alpha}}\end{array}$} & 1-Sustentabilidade financeira da instituição & 4,10 & 1,01 & $24,63 \%$ & $75,46 \%$ \\
\hline & 2-Recolocação custo/benefício da mensalidade & 4,11 & 2,04 & $49,64 \%$ & $72,94 \%$ \\
\hline & 3-Utilização racional dos recursos disponíveis & 4,02 & 1,03 & $25,62 \%$ & $72,25 \%$ \\
\hline & 4-Programas de incentivos & 3,99 & 1,26 & $31,58 \%$ & $69,84 \%$ \\
\hline & Média Geral & 4,06 & 1,03 & $25,37 \%$ & $72,62 \%$ \\
\hline
\end{tabular}

Fonte: dados da pesquisa

A Tabela 4 apresenta diferenças médias aparentemente significativas entre os grupos de alunos e professores em relação ao grupo "Diretor/Coordenador". O grupo Diretor/Coordenador" atribuem importância maior ao indicador referente à sustentabilidade financeira com uma média um pouco superior, aparentemente. O grupo "Alunos" parece atribuir menos importância aos programas de incentivos, em relação aos demais indicadores. Algumas discrepâncias nos resultados podem ser visualizadas no Figura 5.

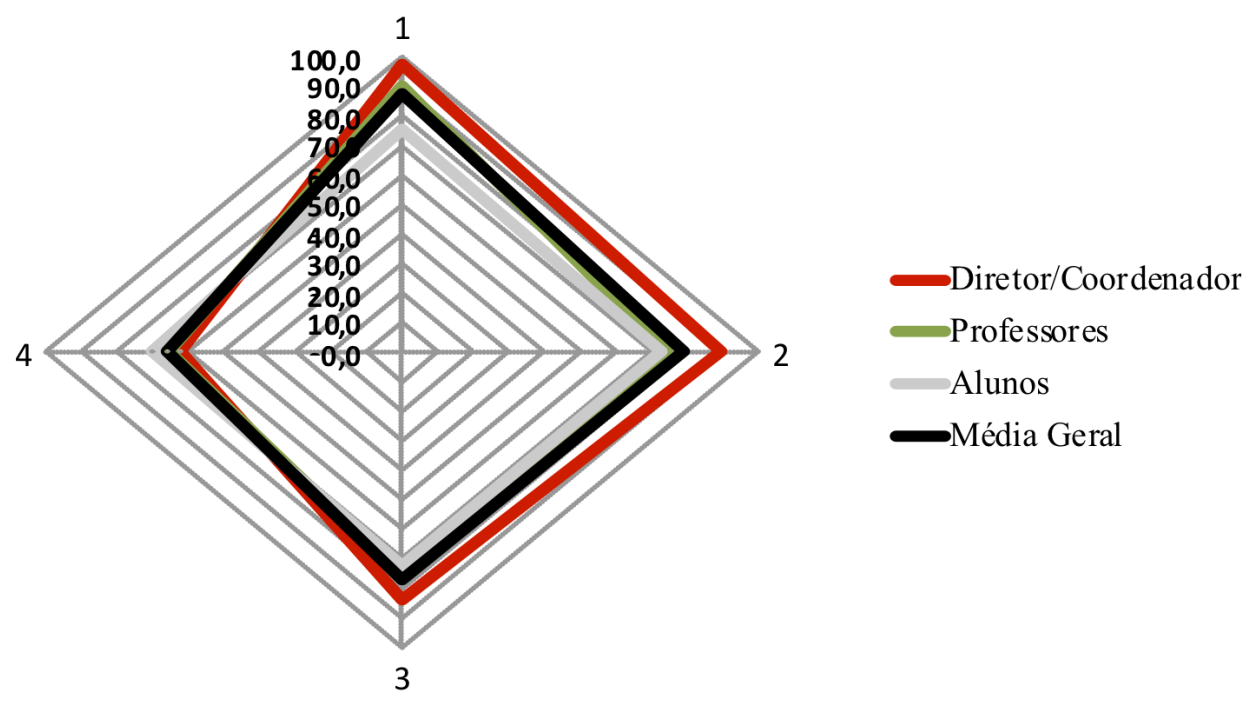

Legenda:

1. Sustentabilidade financeira da instituição

2. Recolocação custo/benefício da mensalidade

3. Utilização racional dos recursos disponíveis

4. Programas de incentivos

Figura 5. Grau de importância para os indicadores da perspectiva Financeira

Fonte: dados da pesquisa 
Pela Figura 5, pôde-se notar uma percepção mais alta do grupo "Diretor/Coordenador" em relação ao grau de importância da perspectiva "Financeira", que parece ter sido afetada pelo indicador sustentabilidade financeira da instituição. No geral, o grupo Diretor/Coordenador atribui maior grau de importância para a maioria dos indicadores.

\subsection{Análise Fatorial para determinação de fatores para as perspectivas do Balanced Scorecard}

O que se pretende mostrar por intermédio da Análise Fatorial é se realmente os 4 indicadores, dentro de cada perspectiva, podem ser representados pelos respectivos fatores que, por sua vez, representam as perspectivas do BSC. Dessa forma, torna-se possível realizar uma análise comparativa para a perspectiva como um todo, em vez de indicador por indicador. O método utilizado foi o de Componentes Principais. A Tabela 5 apresenta os resultados da Análise Fatorial das perspectivas do BSC.

Tabela 5

\section{Análise Fatorial das Perspectivas do BSC (Escala Logarítmica)}

\begin{tabular}{ccccc}
\hline Perspectiva & KMO & Bartlett's & Fatores Extraídos & Variância Explicada \\
\hline Aprendizagem e crescimento & 0,786 & $<0,001$ & 1 & $65,26 \%$ \\
\hline Processos Internos & 0,809 & $<0,001$ & 1 & $68,03 \%$ \\
\hline Clientes & 0,775 & $<0,001$ & 1 & $66,90 \%$ \\
\hline Financeira & 0,813 & $<0,001$ & 1 & $68,70 \%$ \\
\hline
\end{tabular}

Fonte: dados da pesquisa

Pelos resultados da Tabela 5, concluiu-se que os indicadores podem ser reduzidos por um único fator para todas as perspectivas. Os valores da estatística de Kaiser Meyer Olkin são todos bem superiores a 0,600 , o que indica um bom ajuste dos dados ao modelo de análise fatorial. A variância explicada também pode ser considerada bastante razoável, ou seja, os fatores extraídos para cada perspectiva conseguem explicar a variabilidade dos dados por intermédio dos indicadores de Balanced Scorecard estabelecidos. O teste de Bartlett's (Bartlett's Test of Sphericity) comprova que a matriz de correlação entre os indicadores para cada um dos fatores não é uma matriz identidade. Sendo assim, existem correlações significativas entre os indicadores que compõem cada fator determinado. De acordo com os critérios estatísticos apresentados na Tabela 5, as Análises Fatoriais realizadas foram adequadas. Os autovalores referentes às importâncias dos fatores gerados pela análise fatorial conseguiram explicar mais de $65 \%$ da variabilidade dos dados para todas as perspectivas.

\subsubsection{Resultado da comparação média entre os escores calculados pela análise fatorial para as perspectivas do BSC}

De acordo com Tabela 6, para valores menores que 0,05 rejeita-se a hipótese de igualdade de variância entre os grupos. Dessa forma, realizou-se o teste de Scheffe, para verificar para quais grupos existe diferença. 
Tabela 6

Teste de Levene para Igualdade de Variância (Escala Logarítmica)

\begin{tabular}{lccc}
\hline \multicolumn{1}{c}{ Fatores } & Estatística de Levene & Valor $\mathbf{P}$ & Homogeneidade de variância \\
\hline Fator Perspectiva Aprendizagem e Crescimento & 13,799 & 0,000 & Não assumida \\
\hline Fator Perspectiva Processos Internos & 27,592 & 0,000 & Não assumida \\
\hline Fator Perspectiva Clientes & 3,930 & 0,020 & Não assumida \\
\hline Fator Perspectiva Financeira & 18,161 & 0,000 & Não assumida \\
\hline
\end{tabular}

Fonte: dados da pesquisa

A análise que comparou os escores médios dos fatores entre os grupos de análise se mostrou importante para uma análise mais geral acerca do Balanced Scorecard. Confirmou-se uma percepção de menor importância para os entrevistados do grupo de alunos e uma maior importância atribuída aos entrevistados do grupo de coordenadores/diretores, em alguns casos, equiparáveis em relação ao grupo dos professores.

Entretanto, para uma análise mais criteriosa, parece ser mais relevante uma visão dos próprios indicadores, uma vez que ela permite avaliar pontualmente quais os gaps ou diferenças são mais relevantes. A análise por fatores tende a não permitir que diferenças significativas entre os grupos sejam observadas, uma vez que o índice gerado sofre maiores influências de certas variáveis em relação a outras. O mesmo pode ocorrer com a análise da média geral da perspectiva. Em alguns casos, não se observa diferença na média geral, mas existem alguns gaps quando os indicadores são analisados de forma isolada.

O resultado comparativo da média do escore da Análise Fatorial foi semelhante à nota média simples dos indicadores para a perspectiva "Aprendizagem e Crescimento," "Processos Internos" e "Clientes". Para as duas primeiras, a avaliação dos alunos foi inferior em termos de importância. Já para a perspectiva "Clientes", ambas as análises não indicaram diferenças entre os grupos. Entretanto, é mais estratégico verificar os indicadores de forma individual para uma conclusão mais detalhada e mais pontual.

\section{Discussão}

Ao analisar os resultados da pesquisa obtidos por meio da aplicação de questionários, com a direção/coordenação, professores e alunos, para avaliar a consistência estratégica entre esses sujeitos dentro das perspectivas do BSC, observa-se que as percepções entre os stakerolders são diferentes e nem sempre convergem para a mesma opinião.

Ao analisar a percepção referente à "Aprendizagem e Crescimento" e a "Processos Internos", pode-se inferir que os diretores/coordenadores tendem a dar notas mais altas a esses indicadores, no entanto, os alunos tendem a atribuir menos importância a essas perspectivas em relação aos demais grupos. Portanto, considerando que o objetivo da pesquisa é verificar a consistência estratégica na percepção dos stakeholders nas Instituições de Ensino Superior privadas, por meio das perspectivas do Balanced Scorecard, percebe-se, por meio dessa análise, que as percepções são divergentes em alguns atributos. Logo, vai ao encontro de Rocha e de Oliveira Casartelli (2014), que pressupõe que, com a adequação do BSC para as Instituições de Ensino Superior, é possível criar e comunicar a estratégia e as direções, oferecendo aos gestores das IES opções viáveis para decidir seus planos e ações, conforme mostrado na percepção do grupo "Diretor/Coordenador". 
Ao se analisar o grau de importância avaliada sobre o cliente, o grupo de professores indica uma importância um pouco mais baixa em relação aos demais grupos. O Coeficiente de Variação (CV), para três dos quatro indicadores referentes aos percentuais do grau de importância, está inferior aos demais grupos, o que indica uma avaliação bastante negativa. Já quando avaliada a percepção "Financeira", observam-se diferenças médias aparentemente significativas entre os grupos de alunos e professores em relação ao grupo de "diretores/coordenadores". Uma vez que o grupo "Diretor/Coordenador" atribuiu importância maior ao indicador referente à sustentabilidade financeira, com uma média um pouco superior, aparentemente, os alunos parecem atribuir menos importância aos programas de incentivos, em relação aos demais indicadores.

Em relação às análises fatoriais realizadas, percebeu-se que foram adequadas. Os autovalores referentes às importâncias dos fatores gerados pela análise fatorial conseguiram explicar mais de $65 \%$ da variabilidade dos dados para todas as perspectivas.

No geral, confirmou-se uma percepção de menor importância para os entrevistados do grupo "Alunos" e uma maior importância atribuída aos entrevistados do grupo "Coordenador/Diretor", em alguns casos equiparáveis em relação ao grupo dos professores. O mesmo pode ocorrer com a análise da média geral da perspectiva. Em alguns casos, não se observa diferença na média geral, mas existem alguns gaps quando os indicadores são analisados de forma isolada. Sendo assim, é mais estratégico verificar os indicadores de forma individual para uma conclusão mais detalhada e mais pontual. Assim, os resultados enontrados convergem para a literatura pois verificam a importância dos stakeholders por meio da adaptação das quatro perspectivas do BSC, conduzindo-os a uma gestão focada na eficiência e na conscistênia estratégia (Silva, 2009).

\section{Conclusão}

O presente trabalho teve como objetivo geral verificar a consistência estratégica na percepção dos stakeholders nas Instituições de Ensino Superior privadas, por meio das perspectivas do Balanced Scorecard.

Nesse sentido, pode-se inferir que a percepção dos stakeholders são, em geral, diferentes, porém, importantes para a consistência estratégica das IES e seus planejamentos estratégios futuros. Infere-se então que o grupo "Coordenador/Diretor" avalia com uma percepção maior do que os outros grupos analisados. Mas cada grupo por meio dos resultados é capaz de apresentar sua percepção e, a partir delas, as podem empresas verificar sua consistência estratégica a ser desenvolvida dentro da IES, o que torna as percepções por meio do BSC eficientes para as IES, pois elas ajudam a alinhar os interesses dos indivíduos, ou o próprio indivíduo com as estratégias da instituição.

Ao verificar a gestão em IES quanto à investigação da consistência estratégica, que é vista como o compartilhamento de recursos e ações por unidade de negócios, prevendo o nível de desempenho de negócios que podem interferir nos resultados dos alunos, professores e interessados, propondo contribuir para o alinhamento das estratégias e redução dos gaps encontrados, percebe-se que os interesses são diferentes. Logo, isso gera implicações no ambiente estratégico, pois requer uma maior disseminação da metodologia do BSC, participação ativa da alta administração e reconhecimento da importância do planejamento estratégico comum aos interessados.

O BSC é uma ferramenta de auxílio aos gestores para um gestão eficaz e eficiente, o que deve sustentar as IES em momentos de aumentos da concorrência, transformações no setor e exigências governamentais, pois as IES devem aplicar medidas de desempenho apropriadas ao sistema, que reflitam e deem oportunidade de melhorar seu ensino e pesquisa, na qualidade de suas instalações e funcionários. Tais medidas de desempenho também devem incorporar as perspectivas das partes interessadas na IES, as quais devem ser comunicadas a todos e avaliadas constantemente por meio de um método apropriado de gestão, como o BSC, melhorando assim a competitividade das IES, tanto local quanto nacional; também deve-se à limitação de poucas instituições utilizadas, podendo ser estentida a mais instituições e localidades. Apesar de muitas técnicas de gestão utilizadas pelas IES serem baseadas nas solicitações do MEC, vale a pena investigar os benefícios que o Balanced Scorecard como ferramenta de gestão pode alcançar. 
Portanto, considerando os resultados apresentados, a hipótese $\mathrm{H} 1$ foi confirmada, respondendo ao problema de pesquisa de que não existe consistência estratégica interna sob as perspectivas do Balanced Scorecard. Esses resultados têm propósito de contribuir com informações para o processo de gestão e com a consistência das estratégias na visão dos principais interessados da IES.

O trabalho teve por limitação a não segregação dos resultados por instituição, mas uma generalização dos resultados para as todas IES pesquisadas, impossibilitando a compreensão mais detalhada dos dados por IES. Como sugestão de pesquisas futuras tem-se o envolvimento também da parte administrativa das IES, pois os funcionários fazem parte do processo que envolve a gestão institucional, além de lidarem diretamente com as demandas existentes. Como novas pesquisas, pode-se também comparar as estratégias das instituições de ensino do setor público e privado, avaliando melhores práticas por região, apresentando os resultados por instituição, evitando, assim, sua generalização, o que pode facilitar na verificação do alinhamento da percepção das perspectivas e indicadores, considerando a realidade de cada instituição envolvida.

\section{Referências}

Atkinson, A. A.; Waterhouse, J. H. \& Wells, R. B. (1997). A stakeholder approach to strategic performance measurement. MIT Sloan Management Review, 38(3), pp. 25-37

Chen, C. M.; Delmas, M. A. \& Lieberman, M. B. (2015). Production frontier methodologies and efficiency as a performance measure in strategic management research. Strategic Management Journal, 36(1), pp. 19-36. doi: 10.1002/smj.2199.

Clarkson, M. E. (1995). A stakeholder framework for analyzing and evaluating corporate social performance. Academy of management review, 20(1), pp. 92-117. doi: 10.5465/AMR.1995.9503271994.

De Melo Santos, N.; Bronzo, M.; de Oliveira, M. P. V. \& de Resende, P. T. V. (2014). Cultura organizacional, estrutura organizacional e gestão de pessoas como bases para uma gestão orientada por processos e seus impactos no desempenho organizacional. BBR-Brazilian Business Review, 11(3), pp. 106-129. doi: http://dx.doi.org/10.15728/bbr.2014.11.3.5.

Ferreira, J. M. (2013). Perspectivas do Balanced Scorecard na avaliação da consistência estratégica: um estudo baseado na percepção da direção/coordenação e dos alunos em uma Instituição de Ensino Superior. Dissertação de Mestrado, Fundação Instituto Capixaba de Pesquisas em Contabilidade, Economia e Finanças, Vitória, ES, Brasil.

Freeman, R. E. (2010). Strategic management: A stakeholder approach. Cambridge University Press.

Frezatti, F.; Bido, D. S.; Cruz, A. P. C. \& Machado, M. J. C. (2015). A estrutura de artefatos de controle gerencial no processo de inovação: existe associação com o perfil estratégico?. BBR-Brazilian Business Review, 12(1), pp. 129-156. doi: http://dx.doi.org/10.15728/bbr.2015.12.6.

Hair Jr, J. F., Babin, B., Money, A. H., \& Samouel, P. (2005). Fundamentos de métodos de pesquisa em administração. São Paulo: Bookman.

Harrison, J. S.; Hall, E. H. \& Nargundkar, R. (1993). Resource allocation as an outcropping of strategic consistency: Performance implications. Academy of Management Journal, 36(5), pp. 1026-1051. doi: $10.2307 / 256644$.

Hoque, Z. (2014). 20 years of studies on the balanced scorecard: Trends, accomplishments, gaps and opportunities for future research. The British accounting review, 46(1), pp. 33-59. doi: http://dx.doi. org/10.1016/j.bar.2013.10.003.

Kaplan, R. S.; \& Norton, D. P. (1997). A estratégia em ação: Balanced Scorecard. Rio de Janeiro: Campus. 
Kettunem, Juha. (2003). Strategic evaluation of institutions by students in higher education. Perspectives, 7(1), 14-18. DOI: 10.1080/1360310022000056687.

Kettunem, J. (2003). Strategic evaluation of institutions by students in higher education. Perspectives, 7(1), pp. 14-18. doi: 10.1080/1360310022000056687.

Lamberg, J. A.; Tikkanen, H.; Nokelainen, T. \& Suur-Inkeroinen, H. (2009). Competitive dynamics, strategic consistency, and organizational survival. Strategic Management Journal, 30(1), pp. 45-60. doi: $10.1002 /$ smj.726.

Lima Júnior, G. F. de. (2008). Balanced Scorecard como ferramenta de avaliação da consistência estratégica: um estudo em instituição de ensino superior privada. Dissertação de Mestrado, Universidade Federal do Rio Grande do Norte, Natal, RN, Brasil.

Lira, M.; Gonçalves, M. \& Marques, M. D. C. D. C. (2015). Instituições de ensino superior públicas em Portugal: sua administração sob as premissas da new public management e da crise econômica global. Revista Brasileira de Educação, 20(60), pp. 99-119. doi: http://dx.doi.org/10.1590/S1413-24782015206006.

Meneghetti, C.; De Beni, R. \& Cornoldi, C. (2007). Strategic knowledge and consistency in students with good and poor study skills. European Journal of Cognitive Psychology, 19(4-5), pp. 628-649. doi: http://dx.doi.org/10.1080/09541440701325990.

Moore, G. (1999). Tinged Shareholders theory: or what's so special about stakeholders? Busines Ethics: A European Review, 8(2). doi: 10.1111/1467-8608.00136.

Porter, M. E. (1992). Vantagem competitiva (3a ed.). Rio de Janeiro: Campus.

Rocha, D. J. A. (2000). Desenvolvimento do Balanced Scorecard para instituição de ensino superior privada: estudo de caso da unidade 4 da Universidade Gama. Dissertação de Mestrado, Universidade Federal de Santa Catarina, Florianópolis, SC, Brasil.

Rocha, J. M. \& de Oliveira Casartelli, A. (2014). Análise do processo de implantação do Balanced Scorecard (BSC) em uma instituição de ensino superior. Revista Gestão Universitária na América Latina-GUAL, 7(3), pp. 268-290. doi http://dx.doi.org/10.5007/1983-4535.2014v7n3p268.

Rüdell Boligon, J. A., Pentiado Godoy, L., \& Souto Bolzan Medeiros, F. (2014). Balanced Scorecard: Estratégia de Gestão Vinculada à Melhoria da Qualidade Organizacional. GEINTEC-Gestão, Inovação e Tecnologias, 4(3), pp. 1228-1243. doi: 10.7198/S2237-0722201400030017.

Serdar Asan, Ş. \& Tanyaş, M. (2007). Integrating Hoshin Kanri and the balanced scorecard for strategic management: The case of higher education. Total Quality Management, 18(9), pp. 999-1014. doi: $10.1080 / 14783360701592604$.

SPSS Inc. (2003). Advanced Statistical Analysis Using SPSS.

Silva, R. (2009). Balanced Scorecard-BSC-Gestão do Ensino Superior-Gestão profissionalizada e qualidade de ensino para instituições de ensino superior privado. Curitiba: Juruá.

Starik, M. (1994). The Toronto conference: reflections on stakeholders theory. Business and Society, California, 33(1), pp. 82-131. doi: 10.1177/000765039403300105.

Teixeira, J. F. \& de Castro, L. M. (2015). Questões de governança e os papéis dos stakeholders no ambiente do ensino superior brasileiro. Revista Gestão Universitária na América Latina-GUAL, 8(2), pp. 237-257. doi: http://dx.doi.org/10.5007/1983-4535.2015v8n2p237

Venkatraman, N. \& Camillus, J. C. (1984). Exploring the concepts of 'fit' in strategic management. Academy of Management Review, 9(3), pp. 513-525.

Werkema, M. C. C, Aguiar, S. (1996). Planejamento e Análise de Experimentos: como identificar e avaliar os principais indicadores influentes em um processo. Belo Horizonte, MG: Fundação Christiano Otoni, Escola de Engenharia da UFMG. 